\title{
Differentiation and multipotential characteristics of mesenchymal stem cells derived from adipose tissue of an endangered wild cat (Leopardus guigna)
}

\author{
Diana M. Echeverry, Daniela M. Rojas, Constanza J. Aguilera, Daniel M. Veraguas, \\ Joel G. Cabezas, Lleretny Rodríguez-Álvarez, Fidel O. Castro*
}

\begin{abstract}
Adipose tissue derived mesenchymal stem cells (AMSCs) had been isolated and used for cell therapy in domestic cats. For wild cats, the isolation of AMSCs has only been reported in the black-footed cat (Felis nigripes). AMSCs obtained from wild cats may be useful to treat injuries of endangered cat species that remain in captivity or arrive at wildlife rehabilitation centers. Additionally, AMSCs might allow improvement of cloning techniques or assist in derivation of induced pluripotent stem cells. Endangered wild cats such as the guigna (Leopardus guigna), an endemic and endangered species from Chile and Argentina, might benefit greatly from the development of novel treatments or techniques that can be applied for its conservation. The objective of this study was to characterise putative AMSCs from guigna in terms of their main biological attributes, particularly, growth kinetics, differentiation ability and surface marker expression. Results obtained from this characterisation were compared with AMSCs isolated from domestic cats. AMSCs were isolated from peritoneal adipose tissue of female cats and subcutaneous tissue from a female guigna. Migration potential, colony-forming unit assay, mesodermal differentiation and surface marker expression (CD45, CD44, CD90, MHCI and MHCII) were evaluated. Domestic cat and guigna AMSCs displayed similar growth properties in culture. Both AMSC types showed mesodermal differentiation potential, in vitro homing potential and similar surface marker expression. These results indicate that AMSCs from subcutaneous tissue of guigna could have potential use as regenerative treatment for this species and might be considered for use in other biotechnological applications.

Key words: cell therapy, guigna, stem cell, wild cat.
\end{abstract}

\section{INTRODUCTION}

Cell therapy is a promising alternative for the treatment of several pathologies in animal species. For instance, adipose tissue derived from mesenchymal stem cells (AMSCs) has been successfully applied to experimentally treat domestic cats affected by chronic kidney disease, asthma and gingivostomatitis (Quimby et al 2015, Trzil et al 2015, Arzi et al 2016). This treatment was also used to restore elbow functionality in the cougar (Puma concolor) (Gómez et al 2015). Adipose tissue is considered to be the best source of mesenchymal stem cells (MSCs) as it can be accessed with relative ease, a large number of cells can be isolated per gram of tissue and this approach is less invasive than other methods (Martin et al 2002, Kono et al 2014). The collection of tissue samples from wild cats is a difficult task because most species suffer from conservation issues and specimens are rarely available to perform this procedure. Among wild cats, AMSCs have only been isolated from the endangered black-footed cat (Felis nigripes), with cells displaying multilineage differentiation potential and a proliferation rate similar to AMSCs obtained from domestic cats (Gómez et al 2015). The potential of these MSCs is of great relevance for the treatment of endangered species as well as an

Accepted: 12.10 .2018 .

Departmento de Ciencia Animal, Facultad de Ciencias Veterinarias, Universidad de Concepción, Chillán, Chile.

*Corresponding author: F Castro; Avenida Vicente Mendez 595, Chillán, Chile; fidcastro@udec.cl approach to improve reproductive techniques, such as cloning and gamete derivation. The guigna (Leopardus guigna) is a threatened felid species with a distribution restricted only to Chile and Argentina (Napolitano et al 2014). This species is endangered due to its interaction with humans. Many of these individuals get run over on roads or arrive at rehabilitation centers with gunshot wounds or blow trauma. Guigna is a threat to rural poultry producers due to the predation of chickens and eggs (Galvez et al 2013). Recently, this species was affected by large forest fires, arriving at wildlife rehabilitation centers with burns ${ }^{1}$, leading to a significant reduction in the population of guigna. Currently, there are very limited alternatives available for the treatment of wild cat species. The Wildlife Rehabilitation Center at Universidad de Concepción occasionally receives injured guignas that cannot receive proper treatment to improve their health condition. The isolation of AMSCs from guignas could be beneficial for the development of novel techniques for the treatment of wild cats maintained in captivity or rehabilitation centers. The purpose of this study was to isolate and characterise guigna AMSCs and to perform a comparison between AMSCs isolated from guignas and domestic cats.
Facultad de Ciencias Veterinarias y Pecuarias Universidad de Chile. 2017. Fauna silvestre de zonas afectadas huye de los incendios hacia sectores poblados. http://www.veterinaria.uchile.cl/noticias/130676/ fauna-silvestre-de-zonas-afectadas-huye-hacia-sectores-poblados. 


\section{MATERIAL AND METHODS}

\section{SAMPLES}

This study was conducted following standard veterinary practices and with approval of the Ethical Committee for Animal Experimentation of the Universidad de Concepción, permit number: CBE-29-16. Tissue donors were female domestic crossbreed cats (6 to 36 months-old) during routine ovariohysterectomy $(n=3)$. Informed consent was obtained from all cat owners. Subcutaneous adipose tissue from a guigna $(n=1)$ was obtained immediately post-mortem from a 6 month-old female who arrived at the wild animal rescue center. The researchers were not granted permission from regulatory authorities for sampling other tissues of the deceased animal.

\section{ISOLATION OF ADIPOSE MESENCHYMAL STEM CELLS} (AMSCS) FROM CAT AND GUIGNA

Preparation of cultured AMSCs was performed as previously described (Gómez et al 2015) with minor modifications. Adipose tissue was minced and digested in $0.1 \%(\mathrm{w} / \mathrm{v})$ collagenase I (Sigma-Aldrich) at $37^{\circ} \mathrm{C}$ for $20 \mathrm{~min}$. After filtration and centrifugation at $1200 \mathrm{rpm}$ for $5 \mathrm{~min}$, the pellet containing the stromal vascular fraction (SVF) was isolated. Cell number and cell viability were measured by the Luna Automated Cell Counter from Logos Biosystems. The cells ( 2000 cells $\left./ \mathrm{cm}^{2}\right)$ were then plated in $35 \mathrm{~mm}$ culture dishes with HyClone Dulbecco's Modified Eagles Medium (DMEM) supplemented with 50\% fetal bovine serum - FBS (HyClone Fetal Bovine Serum Standard). After 2 days, the medium was changed to $10 \%$ FBS/DMEM/F12. When the cells were confluent (80\%), they were trypsinised and subcultured. Cells were used for differentiation experiments at passages two to three (P2-3). Guigna and domestic cat AMSCs were cultured until P5 to evaluate growth kinetics.

\section{ISOLATION OF SKIN FIBROBLAST FROM CAT AND GUIGNA}

For the establishment of fibroblasts cells from domestic cat and guigna skin samples were taken from the abdominal region of cat females subjected to ovariohysterectomy and from a female guigna post-mortem. The skin samples obtained were cut in small pieces (approximately $1 \mathrm{~mm}^{2}$ ). Once cut, they were placed in $35 \mathrm{~mm}$ plates and allowed to dry for 15 minutes to promote its adherence to the culture dish. Once this was achieved, special media for fibroblasts was added (DMEM/F12 without HEPES, 10\%FBS, 2,4 $\mathrm{mM}$ L-Glutamine, 2,4 $\mathrm{mM}$ sodium piruvate, $10 \mathrm{U} / \mathrm{ml}$ ampicilin/amphotericin, 10ng/ml EGF, $1 \%$ essential and non-essential aminoacids) and the explant was further cultured at $38^{\circ} \mathrm{C}$ with $5 \%$ de $\mathrm{CO}_{2}$ for 7 days. Culture plates were examined microscopically every two days until fibroblasts were seen emerging from the explant.
After reaching $80 \%$ confluence the explant was removed with a sterile forceps and the obtained fibroblasts were expanded in 60 and $100 \mathrm{~mm}$ plates.

\section{GROWTH KINETICS}

The assay was performed as previously described with brief modifications (Kono et al 2014). P1 Cat and guigna AMSCs were plated at a concentration of $\sim 1000 / \mathrm{cm}^{2}$ into $35 \mathrm{~mm}$ dishes and cells were cultured until P5. Cells were counted when they reached confluency in each passage; likewise, the days in which they reached each passage were calculated and growth curves were plotted.

\section{IN VITRO MULTILINEAGE DIFFERENTIATION OF DOMESTIC} CAT AND GUIGNA AMSCS

For differentiation assays, domestic cat and guigna AMSCs at P2-P3 were plated at $~ 8000$ cells $/ \mathrm{cm}^{2}$ in six-well culture dishes containing $3 \mathrm{~mL}$ of DMEM/F12 medium supplemented with $10 \% \mathrm{FBS}$ and cultured at $38.5^{\circ} \mathrm{C}$ under humidified air supplemented with $5 \% \mathrm{CO}_{2}$. When cells reached $70-80 \%$ confluency, the culture medium was replaced with the respective differentiation medium. Adipogenic differentiation was performed as previously described with minor modifications (Gómez et al 2015). The culture medium was replaced with adipogenic induction medium (AIM) consisting of DMEM/F12 medium supplemented with $20 \%$ FBS, $5 \%$ insulin-transferrin-selenium-X (ITS-Gibco), $100 \mathrm{nM}$ dexamethasone, 0.5 $\mathrm{mM}$ 3-isobutyl-1-methylxanthine (IBMX) and $100 \mathrm{mM}$ indometacine and cultured for 3 additional days. At day 3, the AIM was switched to adipogenic maintenance medium (AMM), which consisted of the same components as AIM excluding dexamethasone and IBMX. At day 14, cells were fixed and stained with oil red. Osteogenic differentiation was performed as previously described with minor modifications (Castro et al 2014). The tissue culture medium was replaced with DMEM/F12 supplemented with $10 \%$ FBS, $0.25 \mathrm{mM}$ ascorbic acid, 100 $\mathrm{nM}$ dexamethasone and $10 \mathrm{mM} \beta$-glycerophosphate and cultured for 21 days. Cells were fixed and stained with Alizarin red. Chondrogenic differentiation was performed as previously described with minor modifications (Castro et al 2014). For chondrogenic induction cells were cultured in DMEM/F12 supplemented with 10\% FBS, 4.5 $\mathrm{g} / \mathrm{L}$ D-glucose, $10 \mu \mathrm{L} / \mathrm{mL}$ insulin-transferrin-selenium-X (ITS; Gibco), $100 \mathrm{nM}$ dexamethasone, $1 \mu \mathrm{M}$ ascorbic acid 2-phosphate and $2.5 \%$ equine platelet rich plasma (ePRP) for 30 days. Cells were fixed and stained with Alcian Blue. Cells of control groups were cultured in DMEM/ F12 supplemented with $10 \%$ FBS without inducers or other supplements for exactly the same time periods as experimental groups. All stained cells were visualised with phase-contrast optics using an inverted microscope (Olympus CKX-41). 
MIGRATION ASSAYS

Transwell and scratch assays were used for the migration experiments. Domestic cat and guigna AMSCs at P4 were placed in $6.5 \mathrm{~mm}$ diameter transwell dishes with 8 $\mu \mathrm{m}$ cut-off pore filters (Corning Costar, Cambridge, MA). AMSC were placed on the upper layer of a cell-permeable membrane, and a medium with $1 \%$ ePRP was placed below the cell-permeable membrane as a chemoattractant agent. Medium without FBS was used as control. Incubation period was 3 hours at $38^{\circ} \mathrm{C}, 5 \% \mathrm{CO}_{2}$; the cells that migrated and remained trapped in the membrane were stained and counted. Scratch migration assays were performed with the same mediums. Domestic cat and guigna AMSCs were plated in six-well tissue culture plates and were grown to $80 \%$ confluence and then scratched with a sterile pipette tip to leave a gap of approximately $0.4-0.5 \mathrm{~mm}$ in width. The culture medium was then removed and replaced with fresh culture medium without FBS (control) and a medium with $1 \%$ ePRP. Migration was observed at 0, 2, 4, 8, 24, 48 and 72 hours, and the area was measured with Motic Images 2.0 software. The scratch was drawn freehand in the software to obtain the measurement in $\mathrm{cm}^{2}$ of the area during each of the measurement times.

\section{COLONY-FORMING UNIT ASSAY}

Colony-forming unit (CFU) assays were performed at $\mathrm{P} 1$ on freshly isolated cells at different densities $(250,500$, 1000, 2000, 4000 and 8000 cells $/ 4 \mathrm{~cm}^{2}$ ) as described by Gomez et al (2015) with minor modifications. Cells were plated in 12-well plates in duplicate serial dilutions and cultured in $5 \% \mathrm{CO}_{2}$ and $90 \%$ humidity at $38.5^{\circ} \mathrm{C}$ for two weeks in High glucose DMEM-supplemented medium with $20 \%$ FBS. Then, colonies were fixed with $4 \%$ formalin and stained with $0.5 \%$ crystal violet in methanol for $5 \mathrm{~min}$, washed twice in distilled water and subsequently photographed. Cell clusters of 50 or more cells were considered to be a colony. The rate of colony-forming units-fibroblast (CFU-F) was calculated by dividing the average number of colonies/well by the total number of cells plated/well (Gómez et al 2015).

\section{FLOW CYTOMETRY}

Surface marker expression on domestic cat and guigna AMSCs and domestic cat fibroblasts was assessed by flow cytometry (FCM). Cells at P3-P4 from domestic cat and guigna were incubated with primary mouse anti-CD90 (1:100; cat. no. 14-0909, eBioScience, San Diego, CA, USA), anti-CD45 (1:50; cat. no. MCA2727T, AbD Serotec, Raleigh, NC, USA), anti-CD44 (1:200; SantaCruz Biotechnology), anti-MHCI (1:50; Kingfisher Biotech) and feline anti-MHCII antibodies (1:50; Kingfisher Biotech). Antibody-binding reactions were carried out in a blocking solution of $1 \%$ bovine serum albumin (BSA) and 5\% sheep serum in Dulbecco's Phosphate-Buffered Saline (DPBS) at $4{ }^{\circ} \mathrm{C}$ for one hour. Then, cells were washed in DPBS twice and incubated with secondary sheep anti-mouse fluorescein isothiocyanate (FITC)-conjugated antibody (1:50; cat. no. S3772, Aldrich, St. Louis, MO, USA) for 2 hours at room temperature. Finally, cells were washed with $5 \mathrm{~mL}$ of DPBS to remove excess/unbound antibodies before FCM analysis. Control for nonspecific binding was performed by incubating cells with sheep immunoglobulin $\mathrm{G}(\mathrm{IgG})$ and the secondary conjugated antibody, but not the primary antibody. Unstained cells were used as control for autofluorescence.

\section{RNA EXTRACTION AND QUANTITATIVE RT-PCR ANALYSIS}

The expression of Oct4, Nanog, Cd44, Cd90, Aggrecan, PPAR $\gamma$ and Runx $2 \mathrm{mRNAs}$ were detected by quantitative RT-PCR (qRT-PCR). Feline Sdha (succinate-dehydrogenase-complex-flavoprotein subunit A) housekeeping was used as internal standard. Total RNA was isolated from guigna and domestic cat AMSCs and also from dermal fibroblasts to compare expression. RNA was extracted from each sample using an EZNA RNA extraction kit (Omega, Georgia, USA). The first-strand cDNA was synthesised from $500 \mathrm{ng}$ of DNase-treated total RNA using $50 \mathrm{ng}$ random hexamers (Invitrogen, Waltham, Massachusetts, USA) and 200U of MMLV reverse transcriptase (New England Biolabs Ipswich, Massachusetts, USA) according to the manufacturer's instructions. The primer sequences used for amplification are described in table 1. qRT-PCR amplification was performed in a $10 \mu \mathrm{L}$ reaction mixture for 40 cycles under the following conditions: $94^{\circ} \mathrm{C}$ for $30 \mathrm{~s}, 58^{\circ} \mathrm{C}$ for $30 \mathrm{~s}$ and $72{ }^{\circ} \mathrm{C}$ for $40 \mathrm{~s}$, with additional 7 min incubation at $72{ }^{\circ} \mathrm{C}$ after cycle completion. The data of target genes were plotted as fold changes in relation to the expression level of the housekeeping gene, Sdha. The transcript level of Sdha was used as normalisation control for all analysed samples.

\section{STATISTICAL ANALYSIS}

Data were presented as the mean value \pm standard error mean (SEM) of each cell line for CFU assays. To assess statistical significance of mRNA expression, the Kruskal-Wallis and t-student test were performed to obtain $P$-values using Infostat Software (free version). Additional replicate experiments were also performed when necessary. Differences were considered as significant at $P<0.05$.

\section{RESULTS}

ISOLATION AND GROWTH KINETICS OF AMSCS FROM DOMESTIC CAT AND GUIGNA

AMSCs isolated from domestic cat and guigna adhered to plastic and displayed initial morphological heterogeneity 
Table 1. Primer sequences and qRT-PCR conditions of housekeeping and studied genes used for gene expression analysis in AMSCs and fibroblasts from domestic cat and guigna.

\begin{tabular}{|c|c|c|c|c|}
\hline $\begin{array}{l}\text { Gene } \\
\text { name }\end{array}$ & $\begin{array}{l}\text { Primer sequences } \\
\quad\left(5^{\prime}-3^{\prime}\right)\end{array}$ & $\begin{array}{c}\text { Annealing } \\
\text { temperature }\left({ }^{\circ} \mathrm{C}\right)\end{array}$ & $\begin{array}{l}\text { Product lenght } \\
\text { (bp) }\end{array}$ & $\begin{array}{l}\text { Accession number } \\
\quad(\mathrm{NCBI})\end{array}$ \\
\hline Sdha & $\begin{array}{l}\text { F: GCAGCAGAAGAAGCCATTTG } \\
\text { R: GTCATTGACGGGTCTGTACTC }\end{array}$ & 58 & 103 & XM_003981595.1 \\
\hline Oct4 & $\begin{array}{l}\text { F: CCGAAAGAGAAAGCGAACAAG } \\
\text { R: GACCACATCCTTCTCCAG }\end{array}$ & 55 & 136 & NM_001173441.1 \\
\hline Nanog & $\begin{array}{l}\text { F: CAGCCCCAGATACAGTTACAG } \\
\text { R: GCTGGGCACTAAAATACTTGG }\end{array}$ & 55 & 115 & NM_001009340.1 \\
\hline$C d 44$ & $\begin{array}{l}\text { F: TCGAGGCACCCCATTTCATAGACA } \\
\text { R: ATCAGCTGGCTACTCTGTTGGACT }\end{array}$ & 59 & 128 & XM_011286810.2 \\
\hline Cd90 & $\begin{array}{l}\text { F: AGCACGTGATCTTTGGCACTATGG } \\
\text { R: ACATGTGTACATCCCCTCGTCCTT }\end{array}$ & 59 & 134 & XM_006936729.3 \\
\hline$P P A R \gamma$ & $\begin{array}{l}\text { F: TCGGTTTCAGAAGTGCCTTGCT } \\
\text { R: TGGAGATCTCCGCCAACAGCTTTT }\end{array}$ & 59 & 101 & NM_001113176.1 \\
\hline Sox9 & $\begin{array}{l}\text { F: CGTCAACGAATTCGACCAGTACCT } \\
\text { R: TGCTGTTGATGCCGTAGCTC }\end{array}$ & 60 & 101 & XM_023243815.1 \\
\hline Aggrecan & $\begin{array}{l}\text { F: GCACTGTGGATGTAAGTGGCGAAT } \\
\text { R: ACCCTCCACGAACTCAGAAGTGAT }\end{array}$ & 59 & 104 & XM_023254934.1 \\
\hline
\end{tabular}

with fibroblastic-like appearance. For domestic cat AMSCs, a primary culture with an average of $9.2 \times 10^{4}\left( \pm 2.5 \times 10^{4}\right)$ cells $/ \mathrm{cm}^{2}$ was established. Guigna AMSCs were obtained from an initial cell population of $8.9 \times 10^{4}$ cells extracted from $400 \mathrm{mg}$ of subcutaneous adipose tissue. Cell viability of at least $80 \%$ for both species was demonstrated. Clusters of rapidly expanding cells were observed in guigna AMSCs. The growth curves of domestic cat and guigna AMSCs were similar (figure 1).

\section{COLONY-FORMING UNIT ASSAY}

Domestic cat and guigna AMSCs demonstrated ability to form colonies of 250 to 1000 cells/well. The percentage of CFU decreased when the seeding density was higher than 1000 cells/well for both domestic cat and guigna AMSCs (table 2). However, no significant statistical differences were observed in colony counts for cat AMSCs $(P=0.16)$. No statistical analysis was performed for guigna AMSCs.

\section{FLOW CYTOMETRY}

Flow cytometry analysis (figure 2) showed that both domestic cat and guigna AMSC and domestic cat fibroblast samples were barely positive for stromal marker CD90. Cat fibroblasts were slightly positive for CD45 (2.33\%), MHCI (5.99\%) and MHCII (1.25\%). Domestic cat AMSCs were slightly positive for $\mathrm{CD} 45(1.82 \%)$ and negative for markers CD44, MHCI and MHCII. Guigna AMSCs were slightly positive for CD45 (4.25\%) and CD44 (1.49\%) and were negative for MHCI and MHCII. CD90 expression for domestic cat and guigna AMSCs and fibroblasts was
Table 2. CFU of domestic cat and guigna AMSCs. Cells were seeded at different densities and CFU was calculated in duplicate for each cell line.

\begin{tabular}{lcccc}
\hline $\begin{array}{l}\text { Plating cell } \\
\text { density/well } \\
\left(4 \mathrm{~cm}^{2}\right)\end{array}$ & $\begin{array}{c}\text { CFU guigna } \\
\text { AMSC }\end{array}$ & $\begin{array}{c}\text { \% CFU } \\
\text { guigna } \\
\text { AMSC }\end{array}$ & $\begin{array}{c}\text { CFU cat } \\
\text { AMSC }\end{array}$ & $\begin{array}{c}\text { \% CFU cat } \\
\text { AMSC }\end{array}$ \\
\hline 250 & 45.5 & 18.2 & $7.6 \pm 0.6$ & 3.1 \\
500 & 22.5 & 4.5 & $13.3 \pm 2.5$ & 2.7 \\
1000 & 23.5 & 2.35 & $22.3 \pm 1.5$ & 2.2 \\
2000 & 4.5 & 0.22 & $11 \pm 11.5$ & 0.55 \\
4000 & 21.5 & 0.53 & $21 \pm 10.5$ & 0.52 \\
8000 & 23.5 & 0.29 & $19 \pm 13.1$ & 0.23 \\
\hline
\end{tabular}

similar. Other markers assayed, CD73, 105 and 271, were not detected, most likely due to the species-specific binding of the antibodies (anti-mouse or humans).

\section{IN VITRO MULTILINEAGE DIFFERENTIATION OF DOMESTIC CAT AND GUIGNA AMSCS}

Adipogenic differentiation of domestic cat and guigna AMSCs was indicated by the presence of intracytoplasmic lipid droplets after two week (figures 3 and 4 ) using oil red staining; whereas, control undifferentiated cells showed no lipid deposits. When compared to control, non-differentiated cells, PPA $\gamma$ expression was significantly higher in the guigna $(P=0.0079)$ and cat $(P=0.0039)$ AMSCs differentiated towards the adipogenic lineage (figures 3 and 4). Domestic cat and guigna AMSCs (figures 3 and 4) 


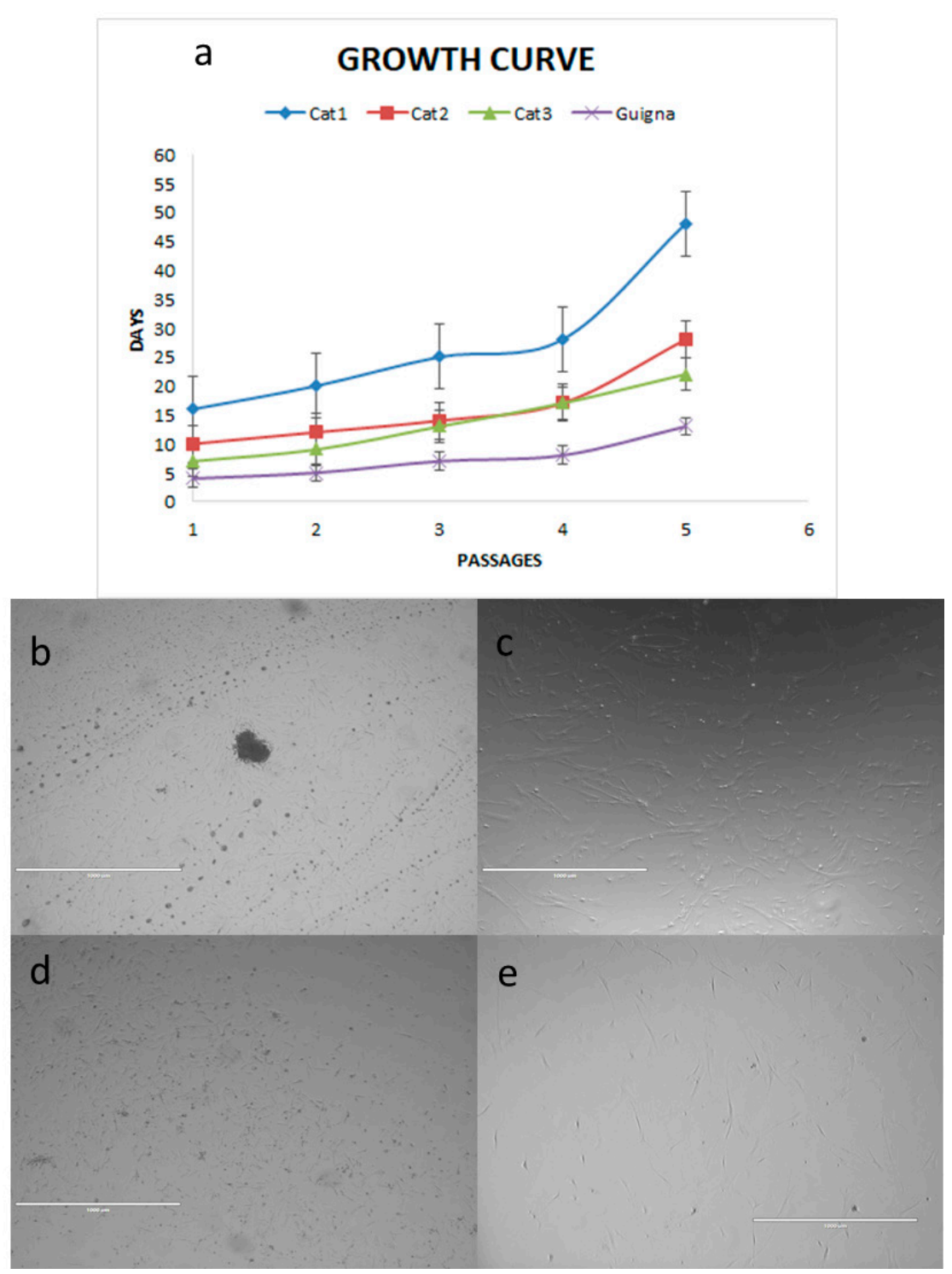

Figure 1. a) Growth curve of cultured domestic cat and guigna AMSCs. Three lines of cat AMSCs and one of guigna AMSCs were cultured until passage 5 (P5) to evaluate growth kinetics; b) AMSCs from guigna at P1 and c) AMSCs from guigna at P5; d) AMSCs from cat at P1 and e) AMSCs from cat at P5. Scale bars represent $1000 \mu \mathrm{m}$.

induced towards osteogenic differentiation for 21 days revealed calcium mineralization after Alizarin red staining and Sox 9 expression significantly higher in cat $(P=0.028)$ and guigna $(P=0.0079)$ AMSC. Osteogenic control of undifferentiated cells were negative for Alizarin red stain (figures 3 and 4). Proteoglycan and glycosaminoglycan depositions following chondrogenic differentiation were shown by Alcian blue staining in domestic cat and guigna AMSCs at day 30 (figures 3 and 4). Chondrogenic control of undifferentiated cells were negative for Alcian blue stain after being cultured with standard medium. The expression of Aggrecan showed a tendency to a higher expression in cat $(P=0.1)$ and guigna $(P=0.16)$ AMSCs with respect to the control group, however there was no significant statistical difference (figures 3 and 4).

\section{MIGRATION ASSAYS}

Transwell and scratch assays proved to be similar for assessing cellular migration. There was a significant increase in migration in the presence of attractant versus non-treated controls in transwell assays for domestic cat $\operatorname{AMSCs}(P=0.03)$ (figure $5 \mathrm{~d}$ ). A greater number of guigna AMSCs migrated through the transwell; however, it was not statistically significant (figure 5d). To complement the results obtained in the transwell assay, it was decided to perform the scratch migration test at different times. AMSCs from domestic cat and guigna migrated and filled the scratch gap efficiently after 48 hours of exposure to $1 \%$ ePRP (figure 5a-c). 

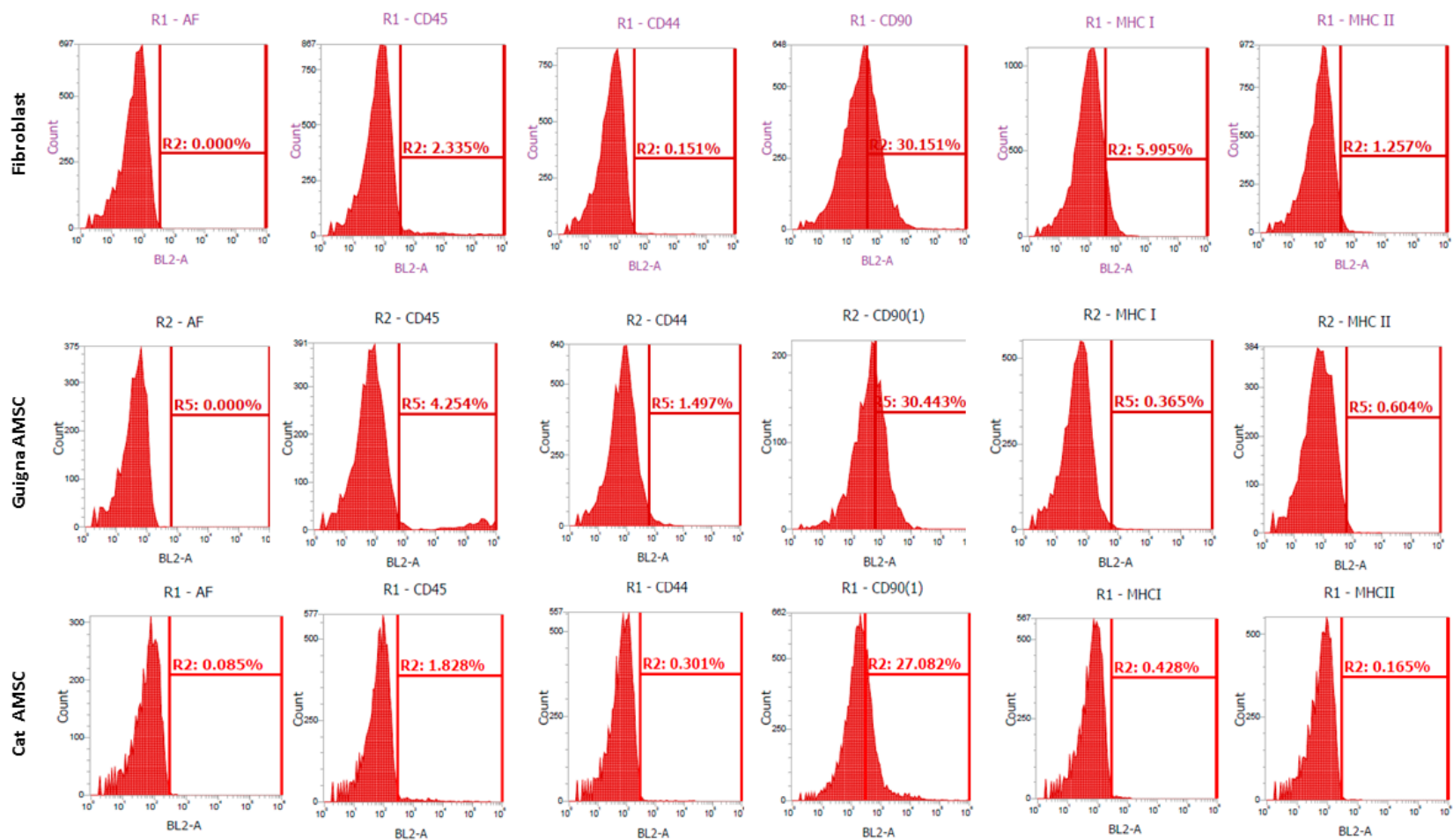

Figure 2. Surface marker expression for domestic cat and guigna AMSCs and for domestic cat fibroblasts. There was a positive expression of CD90 surface marker for fibroblast and AMSCs from domestic cat and guigna. A low percentage of expression was detected in domestic cat fibroblasts and domestic cat and guigna AMSCs for CD45. Guigna AMSCs had a low percentage of positivity for CD44. For domestic cat fibroblasts, MHCI and MHCII expressions were low. For both domestic cat and guigna AMSCs, MHCI and MHCII expressions were 0.16 and $0.6 \%$ respectively. $\mathrm{AF}=$ Autofluorescence, BL2A = Fluorescence channel associated with the blue laser, $\mathrm{R} 1-\mathrm{R} 2=$ Regions of a specific group of cells in the histogram that were analysed.

\section{QUANTITATIVE RT-PCR ANALYSIS}

The qRT-PCR analysis revealed that cat cell lines expressed OCT4, NANOG, CD44, and CD90 (figure 6). There was a significant difference in the expression of NANOG $(P=0.007)$ and CD44 $(P=0.02)$ between cat AMSCs and fibroblast (figure 6a). Testicular tissue containing primordial germ cells was used as internal control (figure 6a). AMSC and dermal fibroblasts from guigna were analysed by RT-PCR to determinate if some of the genes of interest were expressed in this specie. Expression of OCT4, NANOG, CD44 and CD90 was detected in guigna AMSCs. NANOG, CD44 and CD90 were expressed as well in guigna fibroblast (figure $6 \mathrm{~b}$ ).

\section{DISCUSSION}

In the present study, AMSCs were successfully obtained from guigna for the first time. The isolation of AMSCs from subcutaneous and abdominal tissue of cats had been previously reported (Kono et al 2014, Gómez et al 2015). Results for the growth kinetics of domestic cat and guigna AMSCs were consistent with other reports in human and cat (Kern et al 2006, Kim et al 2015, Gómez et al 2015).
Colony-forming unit-fibroblast (CFU-F) was similar for domestic cat and guigna AMSCs and 250 cells seeded in $4 \mathrm{~cm}^{2}$ were enough to form colonies. When initial plating densities were above 2000 cells $/ 4 \mathrm{~cm}^{2}$, the efficiency of $\mathrm{CFU}$ formation was diminished. Similar results were published before for domestic cat AMSCs (Kono et al 2014, Gómez et al 2015). Authors found that the cell growth was not affected by cell contact inhibition in small cell clusters and they continued to proliferate until covering $100 \%$ of the dish surface. Moreover, it has been described that cell proliferation of cat AMSCs plated at a density of $1000 \mathrm{cell} / \mathrm{s} / \mathrm{cm}^{2}$ formed colonies with the highest frequency rate per number of cells plated compared to those plated at cell densities $>4000$ cells $/ \mathrm{cm}^{2}$, and CFU was constant and similar at plating densities $<4000$ cells $/ \mathrm{cm}^{2}$ (Gómez et al 2015).

CD90 was detected in flow cytometry analysis of both domestic cat and guigna AMSCs and fibroblasts; this is consistent with another report (Mumaw et al 2015). Previous descriptions have mentioned that fibroblasts express certain surface markers of MSCs (Halfon et al 2011). Interestingly in humans, it has been shown that the canonical markers of adult MSCs such as CD105, CD166, CD90, CD44, CD29, CD73 and CD9 are also expressed 


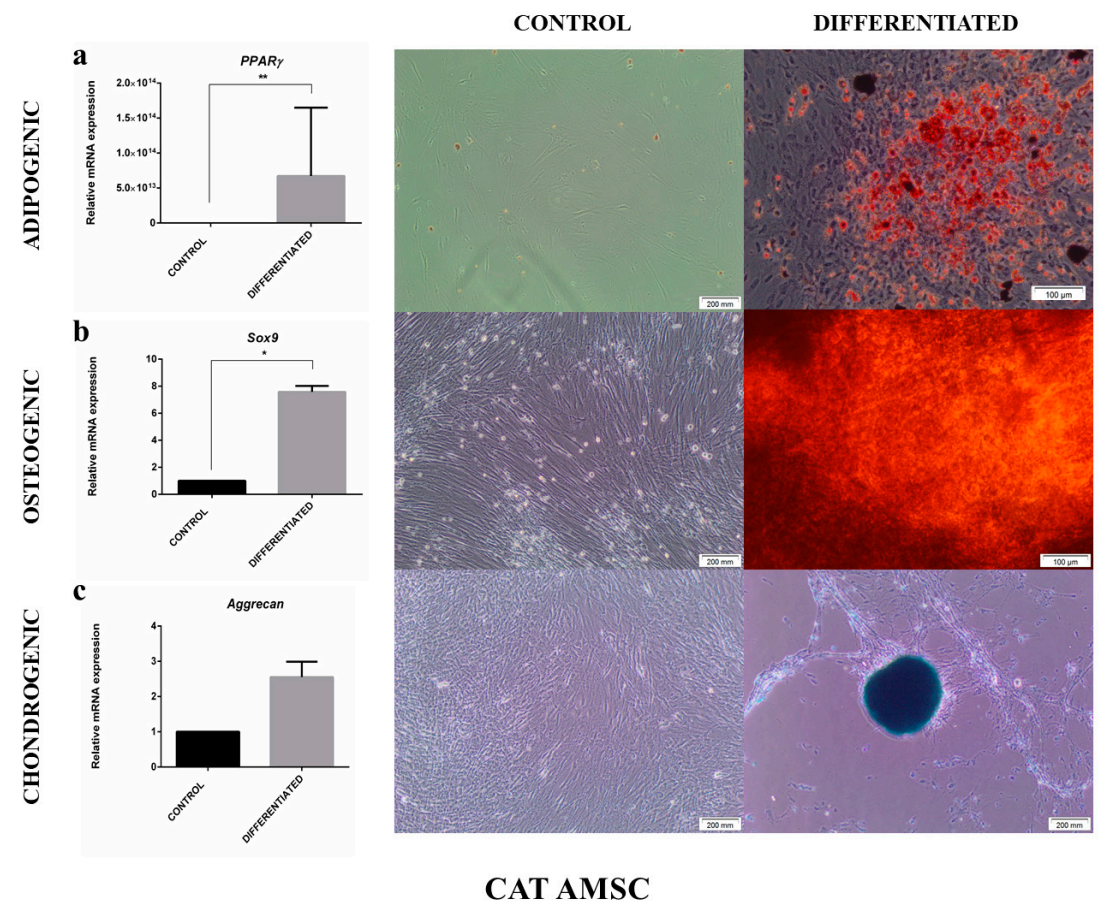

Figure 3. In vitro multilineage differentiation of domestic cat AMSCs. a) Undifferentiated and differentiated domestic cat AMSCs at day 14 of adipogenic differentiation and PPAR $\gamma$ expression. After oil red staining at day 14 intracellular lipids were visualized; b) undifferentiated and differentiated domestic cat AMSCs at day 21 of osteogenic differentiation and Sox 9 expression, the presence of calcium deposits was detected by Alizarin red; c) undifferentiated and differentiated domestic cat AMSCs at day 30 of chondrogenic differentiation and Aggrecan expression. Glycosaminoglycans and proteoglycans were observed in induced domestic cat AMSC at day 30 after Alcian blue staining.
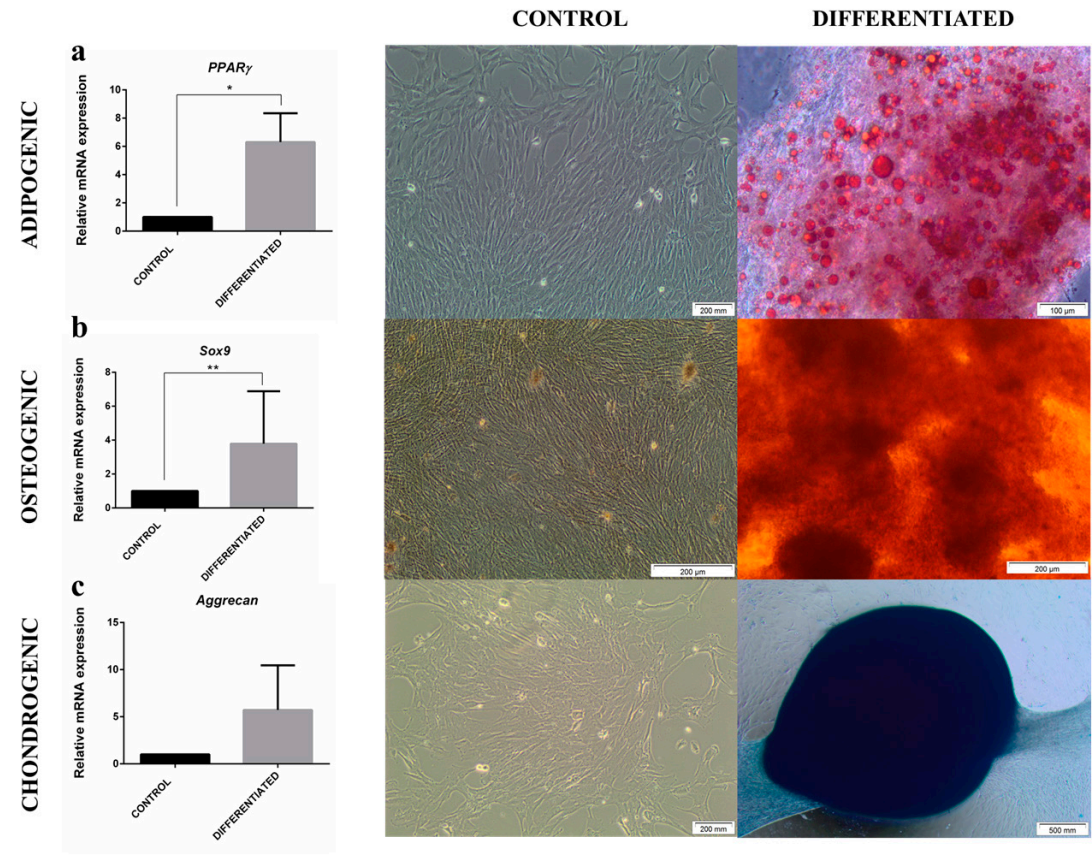

\section{GUIGNA AMSC}

Figure 4. In vitro multilineage differentiation of guigna AMSCs. a) Undifferentiated and differentiated guigna AMSCs at day 14 of adipogenic differentiation and PPAR $\gamma$ expression. After oil red staining at day 14 intracellular lipids were visualized; b) undifferentiated and differentiated guigna AMSCs at day 21 of osteogenic differentiation and Sox 9 expression, the presence of calcium deposits was detected by Alizarin red; c) undifferentiated and differentiated guigna AMSCs at day 30 of chondrogenic differentiation and Aggrecan expression. Glycosaminoglycans and proteoglycans were observed in induced AMSC at day 30 after Alcian blue staining. 

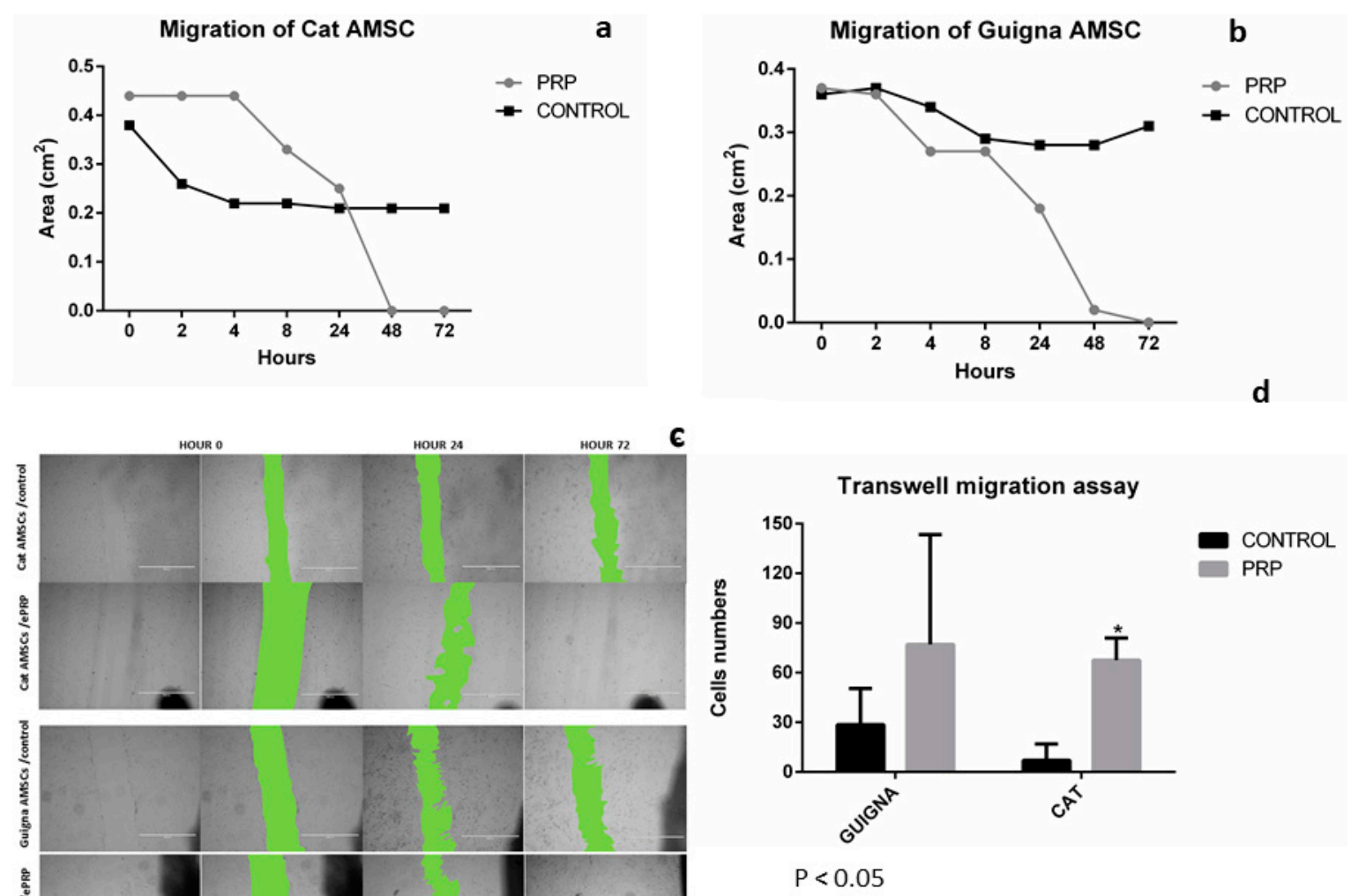

Figure 5. Migration assays. Figures a-c correspond to scratch migration assays. Guigna and domestic cat AMSCs with $1 \%$ ePRP or control medium were photographed at $0,2,4,8,12,24,48$ and 72 hours (c). The area of migration was measured (a and b). Transwell migration assay was evaluated with $1 \% \mathrm{ePRP}$ and control medium. The test was carried out in triplicate (technical replicates). Migrated cells were counted after 2 hour (d). Scale bars represent $1000 \mu \mathrm{m}$. (*) There was a significant statistical difference between the control and treatment with ePRP in the AMSC of domestic cat.

in skin and lung fibroblasts, but this expression decreases with passages (Halfon et al 2011). For Felidae in general, there are no surface markers indicative of stemness. The results of this study confirm that the commonly used CD antigens for human MSCs are not useful to qualify feline stem cells. Other markers should be pursued if accurate selection is required based on surface proteins. Here, the lack of expression of some surface markers, such as CD44, may be related to the passage number of the MSCs used or to the low specificity of antibodies to feline species. It cannot be ruled out that the cell cultures were actually contaminated with fibroblasts from the surrounding tissues. This has been previously reported in literature; fibroblast contaminating cultures could create a potential obstacle for long-term expansion of MSC cultures due to their senescence and may also result in decreased or complete loss of differentiation potential (Prockop and Olson 2007, Rosland et al 2009). Lack of expression of MHCII in cat AMSCs was found, while cat fibroblasts expressed this marker. A very low expression of MHCII was found in guigna AMSCs. The lack of MHCII expression may grant AMSCs a beneficial effect in allogenic cell therapies due to their low immunogenicity (Rutigliano et al 2013). Although the percentage of expression of this marker was close to zero, more studies are required to determine if the guigna AMSCs are suitable for allogenic treatments.

Although inconclusive, our cytometry studies led us to consider complementary characterisation of the putative guigna MSCs with qRT-PCR to evaluate expression of pluripotency genes and surface markers and to compare them with cat cell lines. These results were similar to those reported by Gómez et al (2015), in which Nanog had a higher expression in cat AMSCs when compared to cat fibroblasts. Regarding surface markers, there was significant expression of $\mathrm{Cd} 44$ in domestic cat fibroblasts despite its lack of detection by flow cytometry. This result reinforces the hypothesis of the low specificity of the antibodies used against feline cells. On the other hand, expression of Oct4, Nanog, $C d 44$ and $C d 90$ was observed in guigna AMSCs and expression of Nanog, Cd44 and Cd90 in guigna fibroblasts; however, it is necessary to refine the methods to evaluate the expression of these genes in this species.

Guigna and domestic cat AMSCs showed multilineage differentiation potential by stain and gene expression, as 

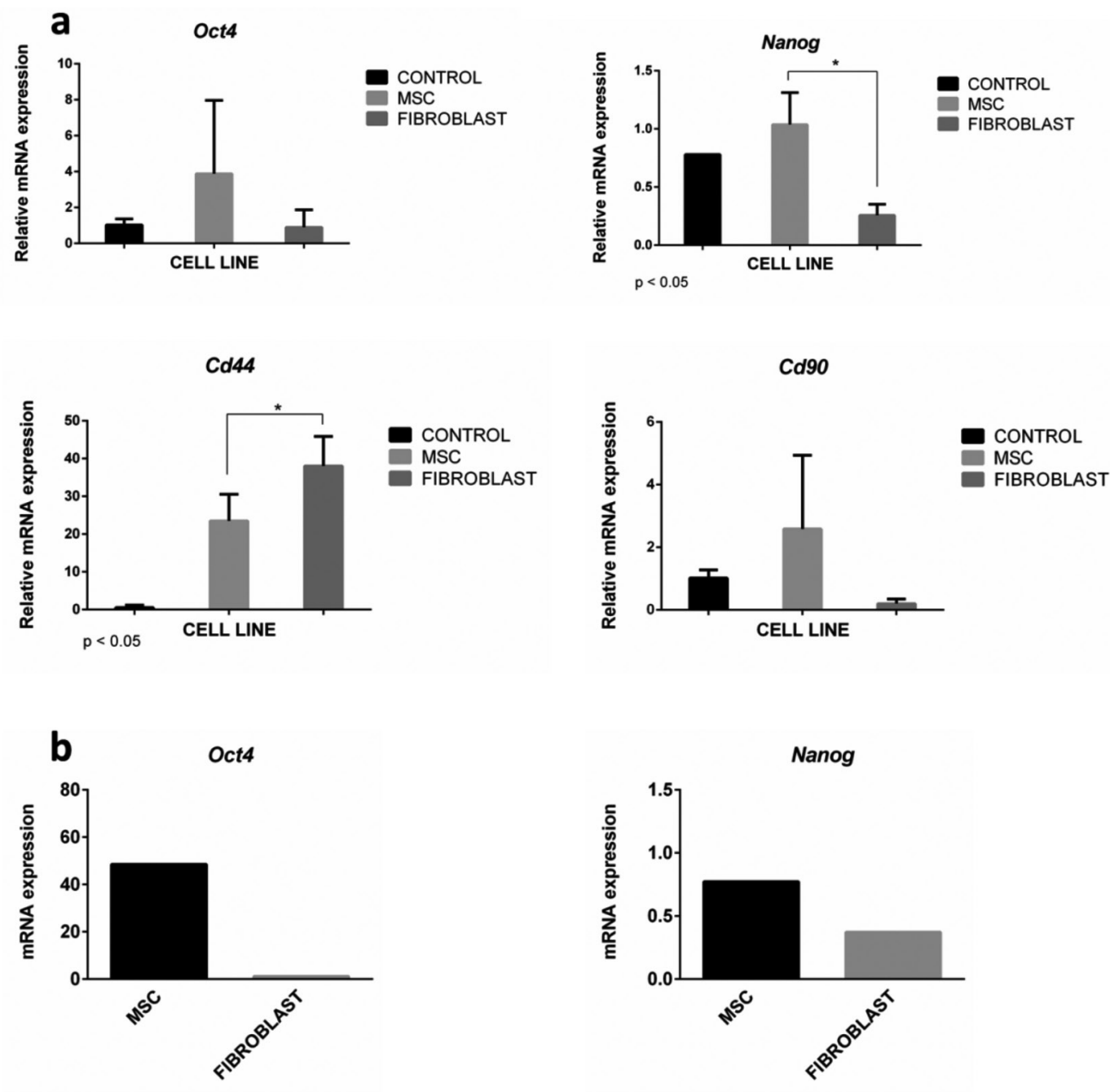

Cd44
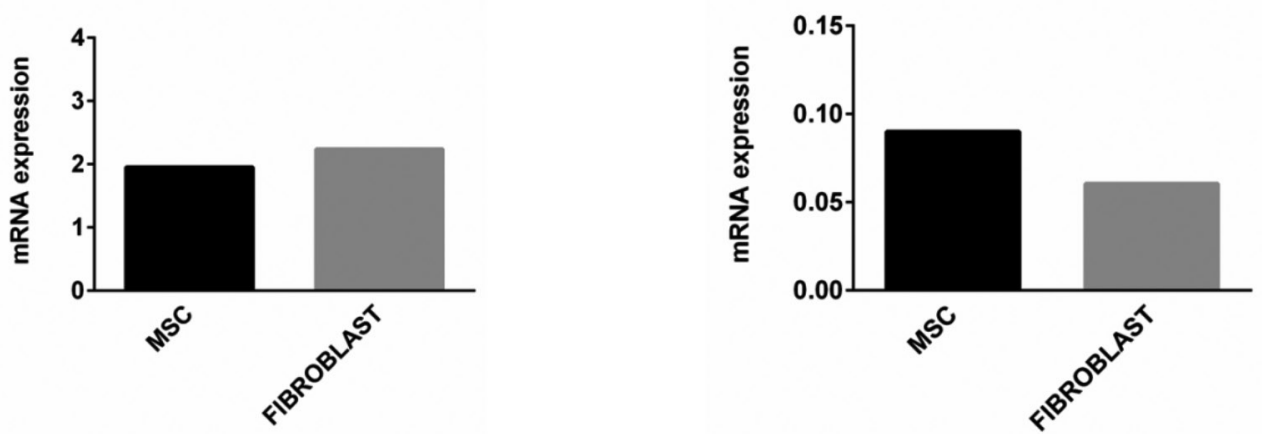

Figure 6. Relative mRNA expression of Oct4, Nanog, $C d 44$ and $C d 90$ in domestic cat AMSCs and fibroblasts (a). Significant differences $(P<0.05)$. Cat testicular tissue was used as comparative control for mRNA expression of Oct4, Nanog, Cd44 and Cd90 domestic cat fibroblasts and AMSCs. Qualitative expression of Oct4, Nanog, Cd44 and Cd90 mRNA for guigna AMSC and fibroblasts (b).

has been previously reported for domestic cats (Kono et al 2014, Gómez et al 2015, Sato et al 2016). For chondrogenic differentiation, in this study ePRP was used instead of TGF-beta with similar results to those previously reported (Castro et al 2014). ePRP is a good source of TGF-beta when used with vitamin $\mathrm{C}$ it as a synergistic action in the up-regulation of Runx2, allowing differentiation towards the chondrogenic lineage (Castro et al 2014).

Homing of cells to the site of the injury is a big issue in cell therapies. Migration assays are used in vitro to 
assess the potential of MSCs to move towards a gradient of chemo attractants, which in turn could be indicative of future homing in vivo. To evaluate the migration potential of domestic cat and guigna AMSCs, we used transwell and scratch assays. In both approaches, domestic cat and guigna AMSCs demonstrated migration potential in medium conditioned with ePRP. Previous reports on the migration ability of domestic cat or guigna MSCs were not found, which makes it difficult to perform a comparison. The choice of PRP as attractant was based on its use in combination with MSCs in some cell therapies. PRP contains important growth factors that act as chemo attractants and mitogens, such as TGF- $\beta 1$, VEGF, PDGF and IGF-1 (Bartold and Raben 1996, Vater et al 2011, Marx et al 2015). In general, the present migration studies yielded similar results to canine AMSCs (Al Delfi et al 2016). Results suggest that guigna AMSCs have migration potential, an important characteristic of multipotent cells. New studies on the potential use of wild-type MSCs are required because of their applicability in cell therapy and potential biotechnological use. Furthermore, other molecular tools are required to allow better analysis of the characteristics of stem cells in non-traditional species.

In this study, guigna AMSCs displayed features of MSCs such as colony formation, differentiation to trilineage and migration towards chemoattractant molecules present in the ePRP. This leads us to propose these cells are MSCs and potential candidates for possible autologous treatments in individuals of this species or to be used in other biotechnological applications, such as cloning. Although more samples of guigna tissue are required in order to confer statistical power to the present study it was showed that, at least in a single isolation of cells from adipose tissue of guigna, there were cells with similar properties as MSC.

\section{ACKNOWLEDGEMENTS}

The authors wish to thank the staff of the Wildlife Rehabilitation Center and the Veterinary Hospital at Universidad de Concepción, Chile. This study was supported by a research grant from Comisión Nacional de Investigación Científica y Tecnológica - CONICYT, Chile (folio 63140147) and funds from the Animal Biotechnology Laboratory, Universidad de Concepción.

\section{REFERENCES}

Arzi B, Mills-Ko E, Verstraete F, Kol A, Walker N, et al. 2016. Therapeutic efficacy of fresh, autologous mesenchymal stem cells for severe refractory gingivostomatitis in cats. Stem Cell Trasl Med 5, 1-12.

Al Delfi IR, Sheard JJ, Wood CR, Vernallis A, Innes JF, et al. 2016. Canine mesenchymal stem cells are neurotrophic and angiogenic: An in vitro assessment of their paracrine activity. Vet J 217, 10-17.

Bartold PM, Raben A. 1996. Growth factor modulation of fibroblasts in simulated wound healing. J Periodontal Res 31, 205-216.
Castro FO, Torres A, Cabezas J, Rodríguez-Álvarez L. 2014. Combined use of platelet rich plasma and vitamin C positively affects differentiation in vitro to mesodermal lineage of adult adipose equine mesenchymal stem cells. Res Vet Sci 96, 95-101.

Gálvez N, Hernández F, Laker J, Gilabert H, Petipas R, et al. 2013. Forest cover outside protected areas plays an important role in the conservation of the Vulnerable guigna Leopardus guigna. Fauna \& Flora International, Oryx, 47, 251-258.

Gómez MC, Qin Q, Biancardi MN, Galiguis J, Dumas C, et al. 2015. Characterization and multilineage differentiation of domestic and black-footed cat mesenchymal stromal/stem cells from abdominal and subcutaneous adipose tissue. Cell Reprogram 17, 376-392.

Halfon S, Abramov N, Grinblat B, Ginis I. 2011. Markers distinguishing mesenchymal stem cells from fibroblasts are downregulated with passaging. Stem Cell Dev 20, 53-66.

Kern S, Eichler H, Stoeve J, Klüter H, Bieback K. 2006. Comparative analysis of mesenchymal stem cells from bone marrow, umbilical cord blood, or adipose tissue. Stem Cells 24, 1294-1301.

Kim H, Lee J, Lee GB, Byeon JS, Gu NY, et al. 2015. Comparison of processing times for isolation of feline adipose tissue-derived mesenchymal stem cells. J Prev Vet Med 39, 101-107.

Kono S, Kazama T, Kano K, Harada K, Uechi M, et al. 2014. Phenotypic and functional properties of feline dedifferentiated fat cells and adipose-derived stem cells. Vet $J$ 199, 88-96.

Martin DR, Cox NR, Hathcock TL, Niemeyer GP, Baker HJ. 2002. Isolation and characterization of multipotential mesenchymal stem cells from feline bone marrow. Exp Hematol 30, 879-886.

Marx C, Silveira MD, Beyer Nardi N. 2015. Adipose-derived stem cells in veterinary medicine: characterization and therapeutic applications. Stem Cells Dev 24, 803-813.

Mumaw JL, Schmiedt CW, Breidling S, Schmiedt CW, Breidling S, et al. 2015. Feline mesenchymal stem cells and supernatant inhibit reactive oxygen species production in cultured feline neutrophils. Res Vet Sci 103, 60-69.

Napolitano C, Johnson WE, Sanderson J, et al. 2014. Phylogeography and population history of Leopardus guigna, the smallest American felid. Conserv Genet 15, 631-653.

Prockop D, Olson S. 2007. Clinical trials with adult stem/progenitor cells for tissue repair: let's not overlook some essential precautions. Blood 109, 3147-3151.

Quimby JM, Webb TL, Randall E, Marolf A, Valdes-Martinez A, et al. 2015. Assessment of intravenous adipose-derived allogeneic mesenchymal stem cells for the treatment of feline chronic kidney disease: a randomized, placebo-controlled clinical trial in eight cats. J Feline Med Surg 1-7.

Rosland G, Svendsen A, Torsvik A, Sobala E. 2009. Long-term cultures of bone marrow-derived human mesenchymal stem cells frequently undergo spontaneous malignant transformation. Cancer Res 69, 5331-5339.

Rutigliano L, Corradetti B, Valentini L, Bizzaro D, Meucci A, et al. 2013. Molecular characterization and in vitro differentiation of feline progenitor-like amniotic epithelial cells. Stem Cell Res Ther 4, 133.

Sato K, Yamawaki-Ogata A, Kanemoto I, Usui A, Narita Y. 2016. Isolation and characterisation of peripheral blood-derived feline mesenchymal stem cells. Vet J 216, 183-188.

Trzil JE, Masseau I, Webb TL, Chang CH, Dodam JR, et al. 2015. Intravenous adipose-derived mesenchymal stem cell therapy for the treatment of feline asthma: a pilot study. J Feline Med Surg 1-10.

Vater C, Kasten P, Stiehler M. 2011. Culture media for the differentiation of mesenchymal stromal cells. Acta Biomater 7, 463-477. 